\title{
Self-Consistent Sources and Conservation Laws for Nonlinear Integrable Couplings of the Li Soliton Hierarchy
}

\author{
Han-yu Wei ${ }^{1,2}$ and Tie-cheng Xia ${ }^{1}$ \\ ${ }^{1}$ Department of Mathematics, Shanghai University, Shanghai 200444, China \\ ${ }^{2}$ Department of Mathematics and Information Science, Zhoukou Normal University, Zhoukou 466001, China
}

Correspondence should be addressed to Han-yu Wei; weihanyu8207@163.com

Received 24 November 2012; Accepted 24 January 2013

Academic Editor: Changbum Chun

Copyright (c) 2013 H.-y. Wei and T.-c. Xia. This is an open access article distributed under the Creative Commons Attribution License, which permits unrestricted use, distribution, and reproduction in any medium, provided the original work is properly cited.

New explicit Lie algebras are introduced for which the nonlinear integrable couplings of the Li soliton hierarchy are obtained. Then, the nonlinear integrable couplings of Li soliton hierarchy with self-consistent sources are established. Finally, we present the infinitely many conservation laws for the nonlinear integrable coupling of Li soliton hierarchy.

\section{Introduction}

Soliton theory has achieved great success during the last decades, it is being applied to many fields. The diversity and complexity of soliton theory enables investigators to do research from different views, such as binary nonlinearization of soliton hierarchy [1] and Bäcklund transformations of soliton systems from symmetry constraints [2].

Recently, with the development of integrable systems, integrable couplings have attracted much attention. Integrable couplings $[3,4]$ are coupled systems of integrable equations, which have been introduced when we study of Virasoro symmetric algebras. It is an important topic to look for integrable couplings because integrable couplings have much richer mathematical structures and better physical meanings. In recent years, many methods of searching for integrable couplings have been developed [5-13], but all the integrable couplings obtained are linear for the $v=$ $\left(v_{1}, \ldots, v_{m}\right)^{T}$. As for how to generate nonlinear integrable couplings, Ma proposed a general scheme [14]. Suppose that an integrable system

$$
u_{t}=K(u)
$$

has a Lax pair $U$ and $V$, which belong to semisimple matrix Lie algebras. Introduce an enlarged spectral matrix

$$
\bar{U}=\bar{U}(\bar{u})=\left[\begin{array}{cc}
U(u) & 0 \\
U_{a}(v) & U(u)+U_{a}(v)
\end{array}\right]
$$

from a zero curvature representation

$$
\overline{U_{t}}-\overline{V_{x}}+[\bar{U}, \bar{V}]=0
$$

where

$$
\bar{V}=\bar{V}(\bar{u})=\left[\begin{array}{cc}
V(u) & 0 \\
V_{a}(\bar{u}) & V(u)+V_{a}(\bar{u})
\end{array}\right],
$$

then we can give rise to

$$
\begin{gathered}
U_{t}-V_{x}+[U, V]=0, \\
U_{a, t}-V_{a, x}+\left[U, V_{a}\right]+\left[U_{a}, V\right]+\left[U_{a}, V_{a}\right]=0 .
\end{gathered}
$$

This is an integrable coupling of (1), and it is a nonlinear integrable coupling because the commutator $\left[U_{a}, V_{a}\right]$ can generate nonlinear terms.

Soliton equation with self-consistent sources (SESCS) [15-22] is an important part in soliton theory. Physically, 
the sources may result in solitary waves with a nonconstant velocity and therefore lead to a variety of dynamics of physical models. For applications, these kinds of systems are usually used to describe interactions between different solitary waves and are relevant to some problems of hydrodynamics, solid state physics, plasma physics, and so forth. How to obtain an integrable coupling of the SESCS is an interesting topic; in this paper, we will use new formula [23] presented by us to generalize soliton hierarchy with self-consistent sources.

The conservation laws play an important role in discussing the integrability for soliton hierarchy. An infinite number of conservation laws for $\mathrm{KdV}$ equation was first discovered by Miura et al. [24]. The direct construction method of multipliers for the conservation laws was presented [25], the Lagrangian approach for evolution equations was considered in [26], Wang and Xia established the infinitely many conservation laws for the integrable super G-J hierarchy [27], and the infinite conservation laws of the generalized quasilinear hyperbolic equations were derived in [28]. Comparatively, the less nonlinear integrable couplings of the soliton equations have been considered for their conservation laws.

This paper is organized as follows. In Section 2, a kind of explicit Lie algebras with the forms of blocks is introduced to generate nonlinear integrable couplings of $\mathrm{Li}$ soliton hierarchy. In Section 3, a new nonlinear integrable coupling of Li soliton hierarchy with self-consistent sources is derived. In Section 4, we obtain the conservation laws for the nonlinear integrable couplings of Li hierarchy. Finally, some conclusions are given.

\section{Lie Algebras for Constructing Nonlinear Integrable Couplings of Li Soliton Hierarchy}

$\mathrm{Tu}$ [29] presented a base of the Li algebra sl(2) as follows:

$$
G_{1}=\operatorname{span}\left\{e_{1}, e_{2}, e_{3}\right\},
$$

where

$$
e_{1}=\left(\begin{array}{cc}
1 & 0 \\
0 & -1
\end{array}\right), \quad e_{2}=\left(\begin{array}{ll}
0 & 1 \\
1 & 0
\end{array}\right), \quad e_{3}=\left(\begin{array}{cc}
0 & 1 \\
-1 & 0
\end{array}\right),
$$

which have the commutative relations

$$
\left[e_{1}, e_{2}\right]=2 e_{2}, \quad\left[e_{1}, e_{3}\right]=-2 e_{3}, \quad\left[e_{2}, e_{3}\right]=e_{1} .
$$

Let us introduce a Lie algebra with matrix blocks by using $G_{1}$ in order to get nonlinear couplings of soliton hierarchy as follows:

$$
G=\operatorname{span}\left\{g_{1}, \ldots, g_{6}\right\}
$$

where

$$
\begin{array}{ll}
g_{1}=\left(\begin{array}{cc}
e_{1} & 0 \\
0 & e_{1}
\end{array}\right), & g_{2}=\left(\begin{array}{cc}
e_{2} & 0 \\
0 & e_{2}
\end{array}\right), \\
g_{3}=\left(\begin{array}{cc}
e_{3} & 0 \\
0 & e_{3}
\end{array}\right), & g_{4}=\left(\begin{array}{cc}
0 & 0 \\
e_{1} & e_{1}
\end{array}\right), \\
g_{5}=\left(\begin{array}{cc}
0 & 0 \\
e_{2} & e_{2}
\end{array}\right), & g_{6}=\left(\begin{array}{cc}
0 & 0 \\
e_{3} & e_{3}
\end{array}\right) .
\end{array}
$$

Define a commutator as follows:

$$
[a, b]=a b-b a, \quad a, b \in G .
$$

A direct verification exhibits that

$$
\begin{gathered}
{\left[g_{1}, g_{2}\right]=2 g_{3}, \quad\left[g_{1}, g_{3}\right]=2 g_{2},} \\
{\left[g_{2}, g_{3}\right]=-2 g_{1}, \quad\left[g_{1}, g_{5}\right]=2 g_{6},} \\
{\left[g_{1}, g_{6}\right]=2 g_{5}, \quad\left[g_{2}, g_{4}\right]=-2 g_{6},} \\
{\left[g_{2}, g_{6}\right]=-2 g_{4},} \\
{\left[g_{3}, g_{4}\right]=-2 g_{5}, \quad\left[g_{3}, g_{5}\right]=2 g_{4},} \\
{\left[g_{4}, g_{5}\right]=2 g_{6}, \quad\left[g_{4}, g_{6}\right]=2 g_{5},} \\
{\left[g_{5}, g_{6}\right]=-2 g_{4}, \quad\left[g_{1}, g_{4}\right]=\left[g_{3}, g_{6}\right]=0 .}
\end{gathered}
$$

Set

$$
\widetilde{G_{1}}=\operatorname{span}\left\{g_{1}, g_{2}, g_{3}\right\}, \quad \widetilde{G_{2}}=\operatorname{span}\left\{g_{4}, g_{5}, g_{6}\right\},
$$

then we find that

$$
G=\widetilde{G_{1}} \oplus \widetilde{G_{2}}, \quad \widetilde{G_{1}} \cong G_{1}, \quad\left[\widetilde{G_{1}}, \widetilde{G_{2}}\right] \subseteq \widetilde{G_{2}},
$$

and $\widetilde{G_{1}}$ and $\widetilde{G_{2}}$ are all simple Lie subalgebras.

While we use Lie algebras to generate integrable hierarchies of evolution equations, we actually employ their loop algebras $\widetilde{G}=G \otimes C\left(\lambda, \lambda^{-1}\right)$ to establish Lax pairs, where $C\left(\lambda, \lambda^{-1}\right)$ represents a set of Laurent ploynomials in $\lambda$ and $G$ is a Lie algebra. Based on this, we give the loop algebras of (9) as follows:

$$
\widetilde{G}=\operatorname{span}\left\{g_{1}(n), \ldots, g_{6}(n)\right\},
$$

where $g_{i}(n)=g_{i} \lambda^{n},\left[g_{i}(m), g_{j}(n)\right]=\left[g_{i}, g_{j}\right] \lambda^{m+n}, 1 \leq i, j \leq 6$, $m, n \in Z$.

We consider an auxiliary linear problem as follows:

$$
\begin{aligned}
& \left(\begin{array}{l}
\varphi_{1} \\
\varphi_{2} \\
\varphi_{3} \\
\varphi_{4}
\end{array}\right)_{x}=U(u, \lambda)\left(\begin{array}{l}
\varphi_{1} \\
\varphi_{2} \\
\varphi_{3} \\
\varphi_{4}
\end{array}\right), \\
& U(u, \lambda)=R_{1}+\sum_{i=1}^{6} u_{i} g_{i}(\lambda), \\
& \left(\begin{array}{l}
\varphi_{1} \\
\varphi_{2} \\
\varphi_{3} \\
\varphi_{4}
\end{array}\right)_{t_{n}}=V_{n}(u, \lambda)\left(\begin{array}{l}
\varphi_{1} \\
\varphi_{2} \\
\varphi_{3} \\
\varphi_{4}
\end{array}\right),
\end{aligned}
$$

where $u=\left(u_{1}, \ldots, u_{s}\right)^{T}, U_{n}=R_{1}+u_{1} g_{1}+\cdots+u_{6} g_{6}, R_{1}$ is a pseudoregular element, $u_{i}(n, t)=u_{i}(i=1,2, \ldots, 6)$, and $\varphi_{i}=$ $\varphi(x, t)$ are field variables defined on $x \in R, t \in R, g_{i}(\lambda) \in \widetilde{G}$.

The compatibility of (16) gives rise to the well-known zero curvature equation

$$
U_{t}-V_{x}+[U, V]=0, \quad \lambda_{t}=0 .
$$


The general scheme of searching for the consistent $V_{n}$, and generating a hierarchy of zero curvature equations was proposed in [30]. Solving the following equation:

$$
\begin{aligned}
& V_{x}=[U, V], \\
& V=\sum_{n=0}^{\infty} V_{n} \lambda^{-n} \\
&=\left(\begin{array}{cccc}
a & b+c & 0 & 0 \\
b-c & -a & 0 & 0 \\
e & f+g & a+e & b+c+f+g \\
f-g & -e & b-c+f-g & -(a+e)
\end{array}\right),
\end{aligned}
$$

then we sesrch for $\triangle_{n} \in \widetilde{G}$, the new $V_{n}$ can be constructed by

$$
V_{n}=\sum_{m=0}^{n} V_{m}(u) \lambda^{n-m}+\Delta_{n}(u, \lambda)
$$

Solving zero curvature (17), we could get evolution equation as follows:

$$
u_{t}=K\left(u, u_{x}, \ldots, \frac{\partial^{p} u}{\partial x^{p}}\right)
$$

Now, we consider Li soliton hierarchy [31]. In order to set up nonlinear integrable couplings of the Li soliton hierarchy with self-consistent sources, we first consider the following matrix spectral problem:

$$
\begin{gathered}
\varphi_{x}=U(u, \lambda) \varphi, \\
U(u, \lambda)=-g_{1}(1)+v g_{1}(0)+u g_{2}(0)+v g_{3}(0) \\
-g_{4}(1)+p_{2} g_{4}(0)+p_{1} g_{5}(0)+p_{2} g_{6}(0),
\end{gathered}
$$

that is,

$$
U(u, \lambda)
$$

$$
\begin{aligned}
& =\left(\begin{array}{cccc}
-\lambda+v & u+v & 0 & 0 \\
u-v & \lambda-v & 0 & 0 \\
-\lambda+p_{2} & p_{1}+p_{2} & -2 \lambda+v+p_{2} & u+v+p_{1}+p_{2} \\
p_{1}-p_{2} & \lambda-p_{2} & u-v+p_{1}-p_{2} & 2 \lambda-v-p_{2}
\end{array}\right) \\
& =\left(\begin{array}{cc}
U_{1} & 0 \\
U_{0} & U_{1}+U_{0}
\end{array}\right),
\end{aligned}
$$

where $\lambda$ is a spectral parameter and $U_{1}$ satisfies $\varphi_{x}=U_{1} \varphi$ which is matrix spectral problem of the Li soliton hierarchy [31].

To establish the nonlinear integrable coupling system of the Li soliton hierarchy, the adjoint equation $V_{x}=[U, V]$ of the spectral problem (21) is firstly solved, we assume that a solution $V$ is given by the following:

$$
\begin{aligned}
V & =\left(\begin{array}{cccc}
a & b+c & 0 & 0 \\
b-c & -a & 0 & 0 \\
e & f+g & a+e & b+c+f+g \\
f-g & -e & b-c+f-g & -(a+e)
\end{array}\right) \\
& =\sum_{n=0}^{\infty} V_{n} \lambda^{-n} \\
& =\sum_{n=0}^{\infty}
\end{aligned}
$$

$$
\times\left(\begin{array}{cccc}
a_{n} & b_{n}+c_{n} & 0 & 0 \\
b_{n}-c_{n} & -a_{n} & 0 & 0 \\
e_{n} & f_{n}+g_{n} & a_{n}+e_{n} & b_{n}+c_{n}+f_{n}+g_{n} \\
f_{n}-g_{n} & -e_{n} & b_{n}-c_{n}+f_{n}-g_{n} & -\left(a_{n}+e_{n}\right)
\end{array}\right) \lambda^{-n} .
$$

Therefore, the condition (18) becomes the following recursion relation:

$$
\begin{gathered}
a_{n, x}=2 v b_{n}-2 u c_{n}, \\
b_{n, x}=-2 c_{n+1}+2 v c_{n}-2 v a_{n}, \\
c_{n, x}=-2 b_{n+1}+2 v b_{n}-2 u a_{n}, \\
e_{n, x}=-2 u g_{n}+2 v f_{n}-2 p_{1} c_{n} \\
+2 p_{2} b_{n}-2 p_{1} g_{n}+2 p_{2} f_{n}, \\
f_{n, x}=-2 g_{n+1}+2 v g_{n}-2 v e_{n} \\
+2 p_{2} c_{n}-2 p_{2} a_{n}+2 p_{2} g_{n}-2 p_{2} e_{n}, \\
g_{n, x}=-2 f_{n+1}+2 v f_{n}-2 u e_{n}+2 p_{2} b_{n} \\
-2 p_{1} a_{n}+2 p_{2} f_{n}-2 p_{1} e_{n} .
\end{gathered}
$$

Choose the initial data

$$
a_{0}=e_{0}=\beta, \quad b_{0}=c_{0}=f_{0}=g_{0}=0,
$$

we see that all sets of functions $a_{n}, b_{n}, c_{n}, e_{n}, f_{n}$, and $g_{n}$ are uniquely determined. In particular, the first few sets are as follows:

$$
\begin{gathered}
a_{1}=0, \quad b_{1}=-u \beta, \quad c_{1}=-v \beta, \quad e_{1}=0, \\
f_{1}=-p_{1} \beta, \quad g_{1}=-p_{2} \beta, \quad a_{2}=\frac{1}{2}\left(v^{2}-u^{2}\right), \\
b_{2}=\left(\frac{1}{2} v_{x}-u v\right) \beta, \quad c_{2}=\left(\frac{1}{2} u_{x}-v^{2}\right) \beta, \\
e_{2}=\left(\frac{1}{2} v p_{2}-\frac{1}{2} u p_{1}+\frac{1}{4} u^{2}-\frac{1}{4} v^{2}-\frac{1}{4} p_{1}^{2}+\frac{1}{4} p_{2}^{2}\right) \beta,
\end{gathered}
$$




$$
\begin{gathered}
f_{2}=\left(\frac{1}{4} p_{2, x}-\frac{1}{4} v_{x}+\frac{1}{2} u v-\frac{1}{2} v p_{1}-\frac{1}{2} u p_{2}-\frac{1}{2} p_{1} p_{2}\right) \beta, \\
g_{2}=\left(\frac{1}{4} p_{1, x}-\frac{1}{4} u_{x}+\frac{1}{2} v^{2}-\frac{1}{2} p_{2}^{2}-v p_{2}\right) \beta, \ldots
\end{gathered}
$$

Considering

$$
V_{n}=V+\triangle_{n}
$$

$\triangle_{n}$

$$
=\left(\begin{array}{cccc}
-\left(a_{n}-c_{n}\right) & 0 & 0 & 0 \\
0 & a_{n}-c_{n} & 0 & 0 \\
-\left(e_{n}-g_{n}\right) & 0 & -\left(a_{n}-c_{n}\right)-\left(e_{n}-g_{n}\right) & \\
0 & e_{n}-g_{n} & 0 & a_{n}-c_{n}+e_{n}-g_{n}
\end{array}\right) \text {. }
$$

From the zero curvature equation $U_{t}-V_{x}+[U, V]=0$, we obtain the nonlinear integrable coupling system

$$
\begin{aligned}
u_{t_{n}} & =K_{n}=\left(\begin{array}{c}
u \\
v \\
p_{1} \\
p_{2}
\end{array}\right)_{t_{n}}=\left(\begin{array}{c}
b_{n, x} \\
-\left(a_{n}-c_{n}\right)_{x} \\
f_{n, x} \\
-\left(e_{n}-g_{n}\right)_{x}
\end{array}\right) \\
& =J\left(\begin{array}{c}
b_{n} \\
a_{n}-c_{n} \\
f_{n} \\
e_{n}-g_{n}
\end{array}\right)=J L^{n}\left(\begin{array}{c}
0 \\
\beta \\
0 \\
\beta
\end{array}\right), \quad n \geq 0,
\end{aligned}
$$

with the Hamiltonian operator $J$ and the hereditary recursion operator $L$, respectively, as follows:

$$
\begin{gathered}
J=\left(\begin{array}{cccc}
\partial & 0 & 0 & 0 \\
0 & -\partial & 0 & 0 \\
0 & 0 & \partial & 0 \\
0 & 0 & 0 & -\partial
\end{array}\right), \\
L=\left(\begin{array}{cccc}
0 & \frac{1}{2} \partial-u & 0 & 0 \\
\partial^{-1} u \partial+\frac{1}{2} \partial & \partial^{-1} v \partial+v & 0 & 0 \\
0 & M_{1} & 0 & M_{2} \\
M_{3} & M_{4} & M_{5} & M_{6}
\end{array}\right),
\end{gathered}
$$

where

$$
\begin{gathered}
M_{1}=-\frac{1}{4} \partial-\frac{1}{2} p_{1}+\frac{1}{2} u, \\
M_{2}=\frac{1}{4} \partial-\frac{1}{2} p_{1}-\frac{1}{2} u, \\
M_{3}=-\frac{1}{2} \partial^{-1} u-\frac{1}{2} \partial^{-1} p_{1}-\partial^{-1} p_{1} \partial-\frac{1}{4} \partial, \\
M_{4}=-\frac{1}{2} \partial^{-1} v \partial+\frac{1}{2} \partial^{-1} p_{2} \partial+\partial^{-1} p_{2} u-\frac{1}{2} v+\frac{1}{2} p_{2}, \\
M_{5}=\frac{1}{2} \partial^{-1} u \partial+\frac{1}{2} \partial^{-1} p_{1} \partial+\frac{1}{4} \partial, \\
M_{6}=-2 \partial^{-1} u v-\frac{3}{2} \partial^{-1} p_{1} v+\frac{1}{2} \partial^{-1} v \partial \\
+\frac{1}{2} \partial^{-1} p_{2} \partial-\frac{1}{2} v+\frac{1}{2} p_{2} .
\end{gathered}
$$

Obviously, when $p_{1}=p_{2}=0$ in (28), the above results become Li soliton hierarchy. So, we can say that (28) is integrable coupling of the Li soliton hierarchy.

Taking $n=2$, we get that the nonlinear integrable couplings of Li soliton hierarchy are as follows:

$$
\begin{gathered}
u_{t_{2}}=\left(-\frac{1}{2} v_{x x}-u_{x} v-u v_{x}\right) \beta, \\
v_{t_{2}}=\left(\frac{1}{2} u_{x x}-3 v v_{x}+u u_{x}\right) \beta, \\
p_{1, t_{2}}=\left(\frac{1}{4} p_{2, x}-\frac{1}{4} v_{x}+\frac{1}{2} u v-\frac{1}{2} v p_{1}\right. \\
\left.-\frac{1}{2} u p_{2}-\frac{1}{2} p_{1} p_{2}\right)_{x} \beta, \\
p_{2, t_{2}=}=\left(\frac{1}{4} p_{1, x}-\frac{1}{4} u_{x}+\frac{1}{2} v^{2}-\frac{3}{4} p_{2}^{2}-\frac{3}{2} v p_{2}\right. \\
\left.+\frac{1}{2} u p_{1}-\frac{1}{4} u^{2}+\frac{1}{4} v^{2}+\frac{1}{4} p_{1}^{2}\right)_{x} \beta .
\end{gathered}
$$

So, we can say that the system in (28) with $n \geq 2$ provides a hierarchy of nonlinear integrable couplings for the Li hierarchy of the soliton equation.

\section{Self-Consistent Sources for the Nonlinear Integrable Couplings of Li Soliton Hierarchy}

According to (16), now we consider a new auxiliary linear problem. For $N$ distinct $\lambda_{j}, j=1,2, \ldots, N$ and the systems of (16) become in the following form:

$$
\begin{aligned}
\left(\begin{array}{l}
\varphi_{1 j} \\
\varphi_{2 j} \\
\varphi_{3 j} \\
\varphi_{4 j}
\end{array}\right)_{x}=U\left(u, \lambda_{j}\right)\left(\begin{array}{l}
\varphi_{1 j} \\
\varphi_{2 j} \\
\varphi_{3 j} \\
\varphi_{4 j}
\end{array}\right) \\
=\sum_{i=1}^{6} u_{i} g_{i}(\lambda)\left(\begin{array}{c}
\varphi_{1 j} \\
\varphi_{2 j} \\
\varphi_{3 j} \\
\varphi_{4 j}
\end{array}\right), \quad j=1, \ldots, N,
\end{aligned}
$$




$$
\begin{aligned}
\left(\begin{array}{l}
\varphi_{1 j} \\
\varphi_{2 j} \\
\varphi_{3 j} \\
\varphi_{4 j}
\end{array}\right)_{t_{n}}= & V_{n}\left(u, \lambda_{j}\right)\left(\begin{array}{l}
\varphi_{1 j} \\
\varphi_{2 j} \\
\varphi_{3 j} \\
\varphi_{4 j}
\end{array}\right) \\
= & {\left[\sum_{m=0}^{n} V_{m}(u) \lambda_{j}^{n-m}+\Delta_{n}\left(u, \lambda_{j}\right)\right] } \\
& \times\left(\begin{array}{c}
\varphi_{1 j} \\
\varphi_{2 j} \\
\varphi_{3 j} \\
\varphi_{4 j}
\end{array}\right), \quad j=1, \ldots, N .
\end{aligned}
$$

Based on the result in [32], we show that the following equation

$$
\frac{\delta H_{k}}{\delta u}+\sum_{j=1}^{N} \alpha_{j} \frac{\delta \lambda_{j}}{\delta u}=0
$$

holds true, where $\alpha_{j}$ is a constant. From (32), we may know that

$$
\begin{aligned}
\frac{\delta \lambda_{j}}{\delta u_{i}} & =\alpha_{j} \operatorname{Tr}\left(\Psi_{j} \frac{\partial U\left(u, \lambda_{j}\right)}{\partial u_{i}}\right) \\
& =\alpha_{j} \operatorname{Tr}\left(\Psi_{j} g_{i}\left(\lambda_{j}\right)\right), \quad i=1,2,
\end{aligned}
$$

where $\operatorname{Tr}$ denotes the trace of a matrix and

$$
\Psi_{j}=\left(\begin{array}{cccc}
\phi_{1 j} \phi_{2 j} & -\phi_{1 j}^{2} & \phi_{3 j} \phi_{4 j} & -\phi_{3 j}^{2} \\
\phi_{2 j}^{2} & -\phi_{1 j} \phi_{2 j} & \phi_{4 j}^{2} & -\phi_{3 j} \phi_{4 j} \\
0 & 0 & \phi_{1 j} \phi_{2 j} & -\phi_{1 j}^{2} \\
0 & 0 & \phi_{2 j}^{2} & -\phi_{1 j} \phi_{2 j}
\end{array}\right),
$$

For $i=3,4$ we define that

$$
\frac{\delta \lambda_{j}}{\delta u_{i}}=\beta_{j} \operatorname{Tr}\left(\Psi_{j A} \frac{\partial U_{0}\left(u, \lambda_{j}\right)}{\partial u_{i}}\right),
$$

where

$$
\begin{gathered}
U=\left(\begin{array}{cc}
U_{1} & 0 \\
U_{0} & U_{1}+U_{0}
\end{array}\right), \\
\Psi_{j A}=\left(\begin{array}{cc}
\phi_{3 j} \phi_{4 j} & -\phi_{3 j}^{2} \\
\phi_{4 j}^{2} & -\phi_{3 j} \phi_{4 j}
\end{array}\right),
\end{gathered}
$$

and $\beta_{j}$ is a constant.

According to (34) and (36), we obtain a kind of nonlinear integrable couplings with self-consistent sources as follows:

$$
\begin{aligned}
u_{t_{n}} & =J \frac{\delta H_{n+1}}{\delta u_{i}}+J \sum_{j=1}^{N} \alpha_{j} \frac{\delta \lambda_{j}}{\delta u} \\
& =J L^{n} \frac{\delta H_{1}}{\delta u_{i}}+J \sum_{j=1}^{N} \alpha_{j} \frac{\delta \lambda_{j}}{\delta u}, \quad n=1,2, \ldots
\end{aligned}
$$

Therefore, according to formulas (34) and (36), we have the following results by direct computations:

$$
\begin{aligned}
& \sum_{j=1}^{N} \frac{\delta \lambda_{j}}{\delta u}=\sum_{j=1}^{N}\left(\begin{array}{c}
\frac{\delta \lambda_{j}}{\delta u} \\
\frac{\delta \lambda_{j}}{\delta v} \\
\frac{\delta \lambda_{j}}{\delta p_{1}} \\
\frac{\delta \lambda_{j}}{\delta p_{1}}
\end{array}\right) \\
& =\left(\begin{array}{c}
2\left(\left\langle\Phi_{2}, \Phi_{2}\right\rangle-\left\langle\Phi_{1}, \Phi_{1}\right\rangle\right) \\
2\left(\left\langle\Phi_{1}, \Phi_{1}\right\rangle+\left\langle\Phi_{2}, \Phi_{2}\right\rangle+2\left\langle\Phi_{1}, \Phi_{2}\right\rangle\right) \\
\left\langle\Phi_{4}, \Phi_{4}\right\rangle-\left\langle\Phi_{3}, \Phi_{3}\right\rangle \\
\left\langle\Phi_{3}, \Phi_{3}\right\rangle+\left\langle\Phi_{4}, \Phi_{4}\right\rangle+2\left\langle\Phi_{3}, \Phi_{4}\right\rangle
\end{array}\right),
\end{aligned}
$$

by taking $\alpha_{j}=1$ and $\beta_{j}=1$ in formulas (34) and (36). Therefore, we have nonlinear integrable coupling system of the Li equations hierarchy with self-consistent sources as follows:

$$
\begin{aligned}
u_{t_{n}}=K_{n} \\
=\left(\begin{array}{c}
u \\
v \\
p_{1} \\
p_{2}
\end{array}\right)_{t_{n}} \\
=J L^{n}\left(\begin{array}{l}
0 \\
\beta \\
0 \\
\beta
\end{array}\right)
\end{aligned}
$$

$$
+J\left(\begin{array}{c}
2\left(\left\langle\Phi_{2}, \Phi_{2}\right\rangle-\left\langle\Phi_{1}, \Phi_{1}\right\rangle\right) \\
2\left(\left\langle\Phi_{1}, \Phi_{1}\right\rangle+\left\langle\Phi_{2}, \Phi_{2}\right\rangle+2\left\langle\Phi_{1}, \Phi_{2}\right\rangle\right) \\
\left\langle\Phi_{4}, \Phi_{4}\right\rangle-\left\langle\Phi_{3}, \Phi_{3}\right\rangle \\
\left\langle\Phi_{3}, \Phi_{3}\right\rangle+\left\langle\Phi_{4}, \Phi_{4}\right\rangle+2\left\langle\Phi_{3}, \Phi_{4}\right\rangle
\end{array}\right)
$$

$$
=J L^{n}\left(\begin{array}{c}
0 \\
\beta \\
0 \\
\beta
\end{array}\right)+J\left(\begin{array}{c}
2 \sum_{j=1}^{N}\left(\varphi_{2 j}^{2}-\varphi_{1 j}^{2}\right) \\
2 \sum_{j=1}^{N}\left(\varphi_{1 j}^{2}+\varphi_{2 j}^{2}+2 \varphi_{1 j} \varphi_{2 j}\right) \\
\sum_{j=1}^{N}\left(\varphi_{4 j}^{2}-\varphi_{3 j}^{2}\right) \\
\sum_{j=1}^{N}\left(\varphi_{3 j}^{2}+\varphi_{4 j}^{2}+2 \varphi_{3 j} \varphi_{4 j}\right)
\end{array}\right) \text {, }
$$


with

$$
\begin{gathered}
\varphi_{1 j, x}=(-\lambda+v) \varphi_{1 j}+(u+v) \varphi_{2 j}, \\
\varphi_{2 j, x}=(u-v) \varphi_{1 j}+(\lambda-v) \varphi_{2 j}, \\
\varphi_{3 j, x}=\left(-\lambda+p_{2}\right) \varphi_{1 j}+\left(p_{1}+p_{2}\right) \varphi_{2 j} \\
+\left(-2 \lambda+v+p_{2}\right) \varphi_{3 j}+\left(u+v+p_{1}+p_{2}\right) \varphi_{4 j}, \\
\varphi_{4 j, x}=\left(p_{1}-p_{2}\right) \varphi_{1 j}+\left(\lambda-p_{2}\right) \varphi_{2 j} \\
+\left(u-v+p_{1}-p_{2}\right) \varphi_{3 j} \\
+\left(2 \lambda-v-p_{2}\right) \varphi_{4 j}, \quad j=1, \ldots, N,
\end{gathered}
$$

where $\Phi_{i}=\left(\varphi_{i 1}, \ldots, \varphi_{i N}\right), i=1,2,3,4$, and $\langle\cdot, \cdot\rangle$ is the standard inner product in $R^{N}$.

When $n=2$ and $\beta=2$, we obtain nonlinear integrable couplings of Li hierarchy with self-consistent sources

$$
\begin{gathered}
u_{t_{2}=}-\frac{1}{2} v_{x x}-u_{x} v-u v_{x}+2 \partial \sum_{j=1}^{N}\left(\varphi_{2 j}^{2}-\varphi_{1 j}^{2}\right), \\
v_{t_{2}}=\frac{1}{2} u_{x x}-3 v v_{x}+u u_{x} \\
-2 \partial \sum_{j=1}^{N}\left(\varphi_{1 j}^{2}+\varphi_{2 j}^{2}+2 \varphi_{1 j} \varphi_{2 j}\right), \\
p_{1 t_{2}}=\left(\frac{1}{4} p_{2, x}-\frac{1}{4} v_{x}+\frac{1}{2} u v-\frac{1}{2} v p_{1}-\frac{1}{2} u p_{2}\right. \\
\left.\quad-\frac{1}{2} p_{1} p_{2}\right)_{x}+\partial \sum_{j=1}^{N}\left(\varphi_{4 j}^{2}-\varphi_{3 j}^{2}\right), \\
p_{2 t_{2}}=\left(\frac{1}{4} p_{1, x}-\frac{1}{4} u_{x}+\frac{1}{2} v^{2}-\frac{3}{4} p_{2}^{2}-\frac{3}{2} v p_{2}+\frac{1}{2} u p_{1}\right. \\
\left.-\frac{1}{4} u^{2}+\frac{1}{4} v^{2}+\frac{1}{4} p_{1}^{2}\right)_{x} \\
-\partial \sum_{j=1}^{N}\left(\varphi_{3 j}^{2}+\varphi_{4 j}^{2}+2 \varphi_{3 j} \varphi_{4 j}\right),
\end{gathered}
$$

with

$$
\begin{gathered}
\varphi_{1 j, x}=(-\lambda+v) \varphi_{1 j}+(u+v) \varphi_{2 j}, \\
\varphi_{2 j, x}=(u-v) \varphi_{1 j}+(\lambda-v) \varphi_{2 j}, \\
\varphi_{3 j, x}=\left(-\lambda+p_{2}\right) \varphi_{1 j}+\left(p_{1}+p_{2}\right) \varphi_{2 j} \\
+\left(-2 \lambda+v+p_{2}\right) \varphi_{3 j}+\left(u+v+p_{1}+p_{2}\right) \varphi_{4 j},
\end{gathered}
$$

$$
\begin{aligned}
\varphi_{4 j, x}= & \left(p_{1}-p_{2}\right) \varphi_{1 j}+\left(\lambda-p_{2}\right) \varphi_{2 j} \\
& +\left(u-v+p_{1}-p_{2}\right) \varphi_{3 j} \\
& +\left(2 \lambda-v-p_{2}\right) \varphi_{4 j}, \quad j=1, \ldots, N .
\end{aligned}
$$

\section{Conservation Laws for the Nonlinear Integrable Couplings of Li Soliton Hierarchy}

In what follows, we will construct conservation laws for the nonlinear integrable couplings of the Li hierarchy. For the coupled spectral problem of Li hierarchy

$U(u, \lambda)$

$$
=\left(\begin{array}{cccc}
-\lambda+v & u+v & 0 & 0 \\
u-v & \lambda-v & 0 & 0 \\
-\lambda+p_{2} & p_{1}+p_{2} & -2 \lambda+v+p_{2} & u+v+p_{1}+p_{2} \\
p_{1}-p_{2} & \lambda+-p_{2} & u-v+p_{1}-p_{2} & 2 \lambda-v-p_{2}
\end{array}\right),
$$

we introduce the variables

$$
M=\frac{\varphi_{2}}{\varphi_{1}}, \quad N=\frac{\varphi_{3}}{\varphi_{1}}, \quad K=\frac{\varphi_{4}}{\varphi_{1}} .
$$

From (44), we have

$$
\begin{gathered}
M_{x}=u-v+2 \lambda M-2 v M-(u+v) M^{2}, \\
N_{x}=-\lambda+p_{2}-\lambda N+\left(p_{1}+p_{2}\right) M \\
+p_{2} N+\left(u+v+p_{1}+p_{2}\right) K-(u+v) N M, \\
K_{x}=p_{1}-p_{2}+3 \lambda K+\lambda M-p_{2} M \\
-\left(2 v+p_{2}\right) K+\left(u-v+p_{1}-p_{2}\right) N-(u+v) K M .
\end{gathered}
$$

We expand $M, N$, and $K$ in powers of $\lambda$ as follows:

$$
\begin{gathered}
M=\sum_{j=1}^{\infty} m_{j} \lambda^{-j}, \quad N=\sum_{j=1}^{\infty} n_{j} \lambda^{-j}, \\
K=\sum_{j=1}^{\infty} k_{j} \lambda^{-j} .
\end{gathered}
$$


Substituting (47) into (46) and comparing the coefficients of the same power of $\lambda$, we obtain the following:

$$
\begin{aligned}
& m_{1}=\frac{1}{2}(v-u), \quad n_{1}=p_{2}, \\
& k_{1}=\frac{1}{3}\left(p_{2}-p_{1}\right)+\frac{1}{6}(u-v) \text {, } \\
& m_{2}=\frac{1}{4}(v-u)_{x}+\frac{1}{2}\left(v^{2}-u v\right) \text {, } \\
& n_{2}=-p_{2, x}-\frac{1}{3} p_{1}^{2}-\frac{2}{3} u p_{1}+\frac{2}{3} v p_{2}+\frac{4}{3} p_{2}^{2}+\frac{1}{6} u^{2}-\frac{1}{6} v^{2}, \\
& k_{2}=\frac{5}{36}(u-v)_{x}+\frac{1}{9}\left(p_{2}-p_{1}\right)_{x}-\frac{5}{18} v^{2}+\frac{5}{18} u v \\
& +\frac{2}{3} v p_{2}-\frac{4}{9} u p_{2}+\frac{4}{9} p_{2}^{2}-\frac{4}{9} p_{1} p_{2}-\frac{2}{9} v p_{1} \\
& m_{3}=\frac{1}{8}(v-u)_{x x}+\frac{1}{8} u^{3}-\frac{1}{8} u^{2} v+\frac{3}{4} v v_{x} \\
& -\frac{1}{2} v u_{x}-\frac{1}{4} u v_{x}+\frac{5}{8} v^{3}-\frac{5}{8} u v^{2} \\
& n_{3}=p_{2, x x}+\frac{5}{9}\left(p_{1} u\right)_{x}-\frac{5}{9}\left(p_{2} v\right)_{x}-\frac{32}{9} p_{2} p_{2, x}-\frac{7}{36} u u_{x} \\
& +\frac{7}{36} v v_{x}+\frac{5}{9} p_{1} p_{1, x}+\frac{1}{9} p_{1} v_{x}-\frac{1}{9} p_{2} u_{x}-\frac{5}{36} u v_{x} \\
& +\frac{1}{9} u p_{2, x}+\frac{5}{36} v u_{x}-\frac{1}{9} v p_{1, x}+\frac{1}{9} p_{1} p_{2, x}-\frac{1}{9} p_{2} p_{1, x} \\
& -\frac{4}{9} p_{1} u v+\frac{11}{9} p_{2} v^{2}-\frac{14}{9} u p_{1} p_{2}+\frac{16}{9} v p_{2}^{2} \\
& +\frac{16}{9} p_{2}^{3}-\frac{7}{9} p_{2} u^{2}-\frac{7}{9} p_{2} p_{1}^{2}+\frac{5}{18} v u^{2}-\frac{5}{18} v^{3}-\frac{2}{9} v p_{1}^{2} \text {, } \\
& k_{3}=\frac{19}{216}(u-v)_{x x}+\frac{1}{27}\left(p_{2}-p_{1}\right)_{x x}+\frac{5}{54}(u-v)_{x} \\
& -\frac{47}{108} v v_{x}+\frac{7}{27} v u_{x}+\frac{19}{108} u v_{x}-\frac{4}{27} v p_{2, x} \\
& +\frac{7}{27} p_{2} v_{x}+\frac{5}{27} u p_{2, x}-\frac{5}{27} p_{2} u_{x}-\frac{5}{27} p_{2} p_{1, x} \\
& +\frac{5}{27} p_{1} p_{2, x}-\frac{2}{27} p_{1} v_{x}-\frac{4}{27} v p_{1, x}-\frac{103}{216} v^{3} \\
& +\frac{101}{216} u v^{2}+\frac{1}{8} v u^{2}+\frac{7}{18} p_{2} v^{2}-\frac{16}{27} p_{2} u v \\
& -\frac{19}{54} p_{2} v^{2}+\frac{32}{27} v p_{2}^{2}-\frac{16}{27} p_{1} p_{2} v-\frac{4}{27} p_{1} v^{2} \\
& -\frac{16}{27} u p_{2}^{2}+\frac{16}{27} p_{2}^{3}-\frac{16}{27} p_{1} p_{2}^{2}+\frac{2}{9} p_{1} u^{2} \\
& +\frac{1}{18} u v^{2}+\frac{1}{3} u p_{1}^{2}+\frac{1}{9} p_{1}^{3}-\frac{2}{9} u v p_{1}-\frac{2}{9} u p_{1} p_{2} \\
& -\frac{1}{9} v p_{1}^{2}-\frac{1}{9} p_{2} p_{1}^{2}-\frac{1}{8} u^{3}, \ldots,
\end{aligned}
$$

and a recursion formula for $m_{j}, n_{j}$, and $k_{j}$ as follows:

$$
\begin{aligned}
m_{j+1} & =\frac{1}{2} m_{j, x}+v m_{j}+\frac{1}{2}(u+v) \sum_{l=1}^{j-1} m_{l} m_{j-l}, \\
n_{j+1}= & -n_{j, x}+\left(p_{1}+p_{2}\right) m_{j}+p_{2} n_{j} \\
& +\left(u+v+p_{1}+p_{2}\right) k_{j}-(u+v) \sum_{l=1}^{j-1} m_{l} n_{j-l}, \\
k_{j+1}= & \frac{1}{3} k_{j, x}-\frac{1}{6} m_{j, x}-\frac{1}{3} v m_{j}+\frac{1}{3} p_{2} m_{j} \\
& +\frac{1}{3}\left(2 v+p_{2}\right) k_{j}-\frac{1}{3}\left(u-v+p_{1}-p_{2}\right) n_{j} \\
& -\frac{1}{6}(u+v) \sum_{l=1}^{j-1} m_{l} m_{j-l}+\frac{1}{3}(u+v) \sum_{l=1}^{j-1} m_{l} k_{j-l} .
\end{aligned}
$$

Because of

$$
\begin{gathered}
\frac{\partial}{\partial t}[-\lambda+v+(u+v) M]=\frac{\partial}{\partial x}[a+(b+c) M] \\
\frac{\partial}{\partial t}\left[-\lambda+p_{2}+\left(p_{1}+p_{2}\right) M+\left(-2 \lambda+v+p_{2}\right) N\right. \\
\left.+\left(u+v+p_{1}+p_{2}\right) K\right] \\
=\frac{\partial}{\partial x}[e+(f+g) M+(a+e) N \\
+(b+c+f+g) K],
\end{gathered}
$$

where

$$
\begin{gathered}
a=\xi_{0} \lambda^{2}+\xi_{1} \lambda+\frac{1}{2} \xi_{0}\left(v^{2}-u^{2}\right), \\
b=-\xi_{0} u \lambda+\xi_{0}\left(-u v+\frac{1}{2} v_{x}\right)-\xi_{1} u, \\
c=-\xi_{0} v \lambda+\xi_{0}\left(-v^{2}+\frac{1}{2} u_{x}\right)-\xi_{1} v, \\
e=\xi_{0} \lambda^{2}+\xi_{1} \lambda \\
+\xi_{0}\left(\frac{1}{2} v p_{2}-\frac{1}{2} u p_{1}+\frac{1}{4} u^{2}-\frac{1}{4} v^{2}-\frac{1}{4} p_{1}^{2}+\frac{1}{4} p_{2}^{2}\right), \\
f=-\xi_{0} p_{1} \lambda+\xi_{0}\left(\frac{1}{4} p_{2, x}-\frac{1}{4} v_{x}+\frac{1}{2} u v-\frac{1}{2} v p_{1}\right. \\
\left.-\frac{1}{2} u p_{2}-\frac{1}{2} p_{1} p_{2}\right)-\xi_{1} p_{1}, \\
g=-\xi_{0} p_{2} \lambda+\xi_{0}\left(\frac{1}{4} p_{1, x}-\frac{1}{4} u_{x}+\frac{1}{2} v^{2}\right. \\
\left.-\frac{1}{2} p_{2}^{2}-v p_{2}\right)-\xi_{1} p_{2} .
\end{gathered}
$$


Assume that

$$
\begin{gathered}
\sigma=-\lambda+v+(u+v) M, \\
\theta=a+(b+c) M, \\
\rho=-\lambda+p_{2}+\left(p_{1}+p_{2}\right) M+\left(-2 \lambda+v+p_{2}\right) N \\
+\left(u+v+p_{1}+p_{2}\right) K, \\
\delta=e+(f+g) M+(a+e) N+(b+c+f+g) K .
\end{gathered}
$$

Then, (50) can be written as $\sigma_{t}=\theta_{x}$ and $\rho_{t}=\delta_{x}$, which are the right form of conservation laws. We expand $\sigma, \theta, \rho$, and $\delta$ as series in powers of $\lambda$ with the coefficients, which are called conserved densities and currents, respectively:

$$
\begin{gathered}
\sigma=-\lambda+v+(u+v) \sum_{j=1}^{\infty} \sigma_{j} \lambda^{-j}, \\
\theta=\xi_{0} \lambda^{2}+\xi_{1} \lambda+\sum_{j=1}^{\infty} \theta_{j} \lambda^{-j}, \\
\rho=-\lambda+p_{2}+\sum_{j=1}^{\infty} \rho_{j} \lambda^{-j}, \\
\delta=\xi_{0} \lambda^{2}+\xi_{1} \lambda+\sum_{j=1}^{\infty} \delta_{j} \lambda^{-j},
\end{gathered}
$$

where $\xi_{0}$ and $\xi_{1}$ are constants of integration. The first conserved densities and currents are read as follows:

$$
\begin{gathered}
\sigma_{1}=\frac{1}{2}\left(v^{2}-u^{2}\right) \\
\theta_{1}=\xi_{0}\left(\frac{1}{2} u u_{x}-\frac{3}{4} u v_{x}-\frac{3}{4} v v_{x}\right)-\frac{1}{2} \xi_{1}\left(v^{2}-u^{2}\right) \\
\rho_{1}=\frac{1}{2} u p_{1}+\frac{1}{3} v p_{2}-\frac{4}{3} p_{2}^{2}-\frac{1}{6} u^{2} \\
+\frac{1}{6} v^{2}+\frac{1}{3} p_{1}^{2}+\frac{1}{6} u p_{1}+2 p_{2, x} \\
\delta_{1}=\xi_{0}\left(2 p_{2, x x}+\frac{1}{36} p_{1} v_{x}+\frac{41}{36} p_{1} u_{x}-\frac{41}{36} p_{2} v_{x}\right. \\
-\frac{1}{36} p_{2} u_{x}-\frac{41}{36} v p_{2, x}-\frac{1}{36} v p_{1, x} \\
+\frac{13}{36} v v_{x}-\frac{1}{36} v u_{x}+\frac{1}{36} u p_{2, x} \\
+\frac{41}{36} u p_{1, x}-\frac{13}{36} u u_{x}-\frac{257}{36} p_{2} p_{2, x} \\
+\frac{41}{36} p_{1} p_{1, x}+\frac{1}{36} p_{1} p_{2, x} \\
-\frac{1}{36} p_{2} p_{1, x}+\frac{47}{36} p_{2} v^{2}-\frac{1}{18} v u^{2}
\end{gathered}
$$

$$
\begin{aligned}
+ & \frac{11}{36} v p_{1} p_{2}+\frac{55}{18} v p_{2}^{2}-\frac{43}{36} p_{2} u^{2} \\
- & \frac{53}{18} u p_{1} p_{2}+\frac{93}{36} p_{2}^{3} \\
- & \frac{3}{4} p_{2} p_{1}^{2}-\frac{1}{18} v p_{1}^{2}-\frac{4}{9} v p_{2}^{2} \\
- & \frac{1}{9} u v p_{1}-\frac{4}{9} p_{1} p_{2}^{2} \\
+ & \left.\frac{1}{18} v^{3}+\frac{1}{36} u v_{x}\right) \\
+\xi_{1}( & -2 p_{2, x}+v p_{1}-\frac{5}{3} u p_{1}+\frac{5}{3} v p_{2}-u p_{2} \\
& \left.+\frac{7}{3} p_{2}^{2}+\frac{1}{6} u^{2}-\frac{1}{6} v^{2}-\frac{1}{3} p_{1}^{2}\right), \ldots
\end{aligned}
$$

The recursion relations for $\sigma_{j}, \theta_{j}, \rho_{j}$, and $\delta_{j}$ are as follows:

$$
\begin{aligned}
& \sigma_{j}=(u+v) m_{j} \\
& \theta_{j}=-\xi_{0}(u+v) m_{j+1}+\xi_{0}\left(\frac{1}{2} u_{x}+\frac{1}{2} v_{x}-u v-v^{2}\right) m_{j} \\
& -\xi_{1}(u+v) m_{j} \\
& \rho_{j}=\left(p_{1}+p_{2}\right) m_{j}-2 n_{j+1}+\left(v+p_{2}\right) n_{j} \\
& +\left(u+v+p_{1}+p_{2}\right) k_{j}, \\
& \delta_{j}=\xi_{0}\left[-\left(p_{1}+p_{2}\right) m_{j+1}\right. \\
& +\left(\frac{1}{4} p_{2, x}+\frac{1}{4} p_{1, x}-\frac{1}{4} v_{x}\right. \\
& -\frac{1}{4} u_{x}+\frac{1}{2} u v-\frac{1}{2} v p_{1} \\
& -\frac{1}{2} u p_{2}-\frac{1}{2} p_{1} p_{2}+\frac{1}{2} v^{2} \\
& \left.-\frac{1}{2} p_{2}^{2}-p_{2} v\right) m_{j} \\
& +2 n_{j+2}+\left(\frac{1}{2} v^{2}-\frac{1}{2} u^{2}+\frac{1}{2} v p_{2}\right. \\
& -\frac{1}{2} u p_{1}+\frac{1}{4} u^{2} \\
& \left.+\frac{1}{4} p_{2}^{2}-\frac{1}{4} v^{2}-\frac{1}{4} p_{1}^{2}\right) n_{j} \\
& -\left(u+v+p_{1}+p_{2}\right) k_{j+1}
\end{aligned}
$$




$$
\begin{gathered}
+\left(\frac{1}{4} u_{x}+\frac{1}{4} v_{x}+\frac{1}{4} p_{1, x}+\frac{1}{4} p_{2, x}-\frac{1}{2} u v\right. \\
-\frac{1}{2} v^{2}-\frac{1}{2} v p_{1}-\frac{1}{2} u p_{2} \\
\left.\left.\quad-\frac{1}{2} p_{1} p_{2}-\frac{1}{2} p_{2}^{2}-v p_{2}\right)\right] \\
+\xi_{1}\left[2 n_{j+1}-\left(p_{1}+p_{2}\right) m_{j}\right. \\
\left.-\left(u+v+p_{1}+p_{2}\right) k_{j}\right]
\end{gathered}
$$

where $m_{j}, n_{j}$, and $k_{j}$ can be calculated from (49). The infinite conservation laws of nonlinear integrable couplings (37) can be easily obtained in (45)-(55), respectively.

\section{Conclusions}

In this paper, a new explicit Lie algebra was introduced, and a new nonlinear integrable couplings of Li soliton hierarchy with self-consistent sources was worked out. Then, the conservation laws of Li soliton hierarchy were also obtained. The method can be used to other soliton hierarchy with selfconsistent sources. In the near future, we will investigate exact solutions of nonlinear integrable couplings of soliton equations with self-consistent sources which are derived by using our method.

\section{Acknowledgments}

The study is supported by the National Natural Science Foundation of China (Grant nos. 11271008, 61072147, and 1071159 ), the Shanghai Leading Academic Discipline Project (Grant no. J50101), and the Shanghai University Leading Academic Discipline Project (A. 13-0101-12-004).

\section{References}

[1] W. X. Ma, B. Fuchssteiner, and W. Oevel, "A $3 \times 3$ matrix spectral problem for AKNS hierarchy and its binary nonlinearization," Physica A, vol. 233, no. 1-2, pp. 331-354, 1996.

[2] W.-X. Ma and X. Geng, "Bäcklund transformations of soliton systems from symmetry constraints," in CRM Proceedings and Lecture Notes, vol. 29, no. 3, pp. 313-323, 2001.

[3] W. X. Ma and B. Fuchssteiner, "Integrable theory of the perturbation equations," Chaos, Solitons and Fractals, vol. 7, no. 8, pp. 1227-1250, 1996.

[4] W.-X. Ma, "Integrable couplings of soliton equations by perturbations. I. A general theory and application to the KdV hierarchy," Methods and Applications of Analysis, vol. 7, no. 1, pp. 21-56, 2000.

[5] T. C. Xia, G. L. Zhang, and E. G. Fan, "New integrable couplings of generalized Kaup-Newell hierarchy and its hamiltonian structures," Communications in Theoretical Physics, vol. 56, no. 1, pp. 1-4, 2011.

[6] Y. Zhang and H. Zhang, "A direct method for integrable couplings of TD hierarchy," Journal of Mathematical Physics, vol. 43, no. 1, pp. 466-472, 2002.
[7] W.-X. Ma, X.-X. Xu, and Y. Zhang, "Semi-direct sums of Lie algebras and continuous integrable couplings," Physics Letters A, vol. 351, no. 3, pp. 125-130, 2006.

[8] G. C. Wu, "New trends in the variational iteration method," Communications in Fractional Calculus, vol. 2, no. 2, pp. 59-75, 2011.

[9] J. Fujioka, "Lagrangian structure and Hamiltonian conservation in fractional optical solitons," Communications in Fractional Calculus, vol. 1, no. 1, pp. 1-14, 2010.

[10] F. Guo and Y. Zhang, "The integrable coupling of the AKNS hierarchy and its Hamiltonian structure," Chaos, Solitons and Fractals, vol. 32, no. 5, pp. 1898-1902, 2007.

[11] H. Jafari, N. Kadkhoda, and C. M. Khalique, "Exact solutions of $\varphi^{4}$ equation using lie symmetry approach along with the simplest equation and Exp-function methods," Abstract and Applied Analysis, vol. 2012, Article ID 350287, 2012.

[12] W.-X. Ma, "Variational identities and applications to Hamiltonian structures of soliton equations," Nonlinear Analysis, vol. 71, no. 12, pp. e1716-e1726, 2009.

[13] W. X. Ma, J. H. Meng, and H. Q. Zhang, "Integrable couplings, variational identities and Hamiltonian formulations," in Proceedings of the 6th International Federation of Nonlinear Analysts Conference, pp. 1-17, 2012.

[14] W.-X. Ma, "Nonlinear continuous integrable Hamiltonian couplings," Applied Mathematics and Computation, vol. 217, no. 17, pp. 7238-7244, 2011.

[15] D. J. Kaup, "Integrable ponderomotive system: cavitons are solitons," Physical Review Letters, vol. 59, no. 18, pp. 2063-2066, 1987.

[16] M. Nakazawa, E. Yamada, and H. Kubota, "Coexistence of self-induced transparency soliton and nonlinear Schrödinger soliton," Physical Review Letters, vol. 66, no. 20, pp. 2625-2628, 1991.

[17] F. J. Yu and L. Li, "Non-isospectral integrable couplings of Ablowitz-Kaup-Newell-Segur (AKNS) hierarchy with selfconsistent sources," Chinese Physics B, vol. 17, no. 11, pp. 39653973, 2008.

[18] T. Xiao and Y. Zeng, "The quasiclassical limit of the symmetry constraint of the KP hierarchy and the dispersionless KP hierarchy with self-consistent sources," Journal of Nonlinear Mathematical Physics, vol. 13, no. 2, pp. 193-204, 2006.

[19] H.-Y. Wang, X.-B. Hu, and H.-W. Tam, "Construction of $q$-discrete two-dimensional Toda lattice equation with selfconsistent sources," Journal of Nonlinear Mathematical Physics, vol. 14, no. 2, pp. 258-268, 2007.

[20] Y.-P. Sun and H.-W. Tam, "New type of nonisospectral KP equation with self-consistent sources and its bilinear Bäcklund transformation," Journal of Nonlinear Mathematical Physics, vol. 18, no. 2, pp. 323-336, 2011.

[21] S. F. Deng, "Multisoliton solutions for the isospectral and nonisospectral BKP equation with self-consistent sources," Chinese Physics Letters, vol. 25, no. 7, pp. 2331-2334, 2008.

[22] S.-f. Deng, "The multisoliton solutions for the nonisospectral mKPI equation with self-consistent sources," Physics Letters A, vol. 372 , no. 4 , pp. 460-464, 2008.

[23] T. C. Xia, "Two new integrable couplings of the soliton hierarchies with self-consistent sources," Chinese Physics B, vol. 19, no. 10, Article ID 100303, pp. 1-8, 2010.

[24] R. M. Miura, C. S. Gardner, and M. D. Kruskal, "Kortewegde Vries equation and generalizations. II. Existence of conservation laws and constants of motion," Journal of Mathematical Physics, vol. 9, no. 8, pp. 1204-1209, 1968. 
[25] S. C. Anco and G. Bluman, "Direct construction method for conservation laws of partial differential equations. II. General treatment," European Journal of Applied Mathematics, vol. 13, no. 5, pp. 567-585, 2002.

[26] N. H. Ibragimov and T. Kolsrud, "Lagrangian approach to evolution equations: symmetries and conservation laws," Nonlinear Dynamics, vol. 36, no. 1, pp. 29-40, 2004.

[27] H. Wang and T.-C. Xia, "Conservation laws for a super GJ hierarchy with self-consistent sources," Communications in Nonlinear Science and Numerical Simulation, vol. 17, no. 2, pp. 566-572, 2012.

[28] M. Nadjafikhah, R. Bakhshandeh Chamazkoti, and F. Ahangari, "Potential symmetries and conservation laws for generalized quasilinear hyperbolic equations," Applied Mathematics and Mechanics, vol. 32, no. 12, pp. 1607-1614, 2011.

[29] G. Z. Tu, "On Liouville integrability of zero-curvature equations and the Yang hierarchy," Journal of Physics A, vol. 22, no. 13, pp. 2375-2392, 1989.

[30] G. Z. Tu, "The trace identity, a powerful tool for constructing the Hamiltonian structure of integrable systems," Journal of Mathematical Physics, vol. 30, no. 2, pp. 330-338, 1989.

[31] Y.-S. Li, "The reductions of the Darboux transformation and some solutions of the soliton equations," Journal of Physics A, vol. 29, no. 14, pp. 4187-4195, 1996.

[32] G. Z. Tu, "An extension of a theorem on gradients of conserved densities of integrable systems," Northeastern Mathematical Journal, vol. 6, no. 1, pp. 28-32, 1990. 


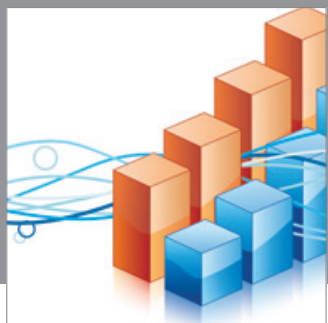

Advances in

Operations Research

mansans

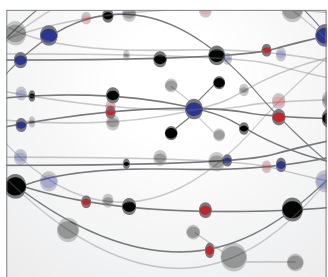

The Scientific World Journal
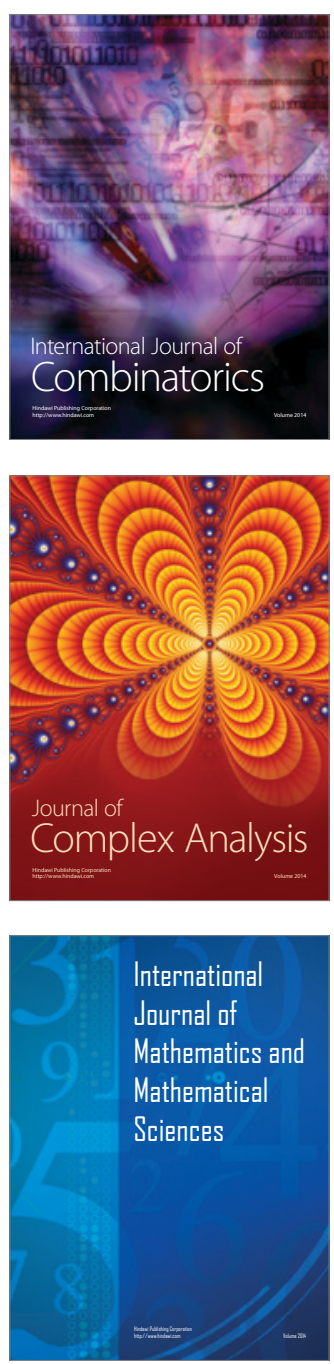
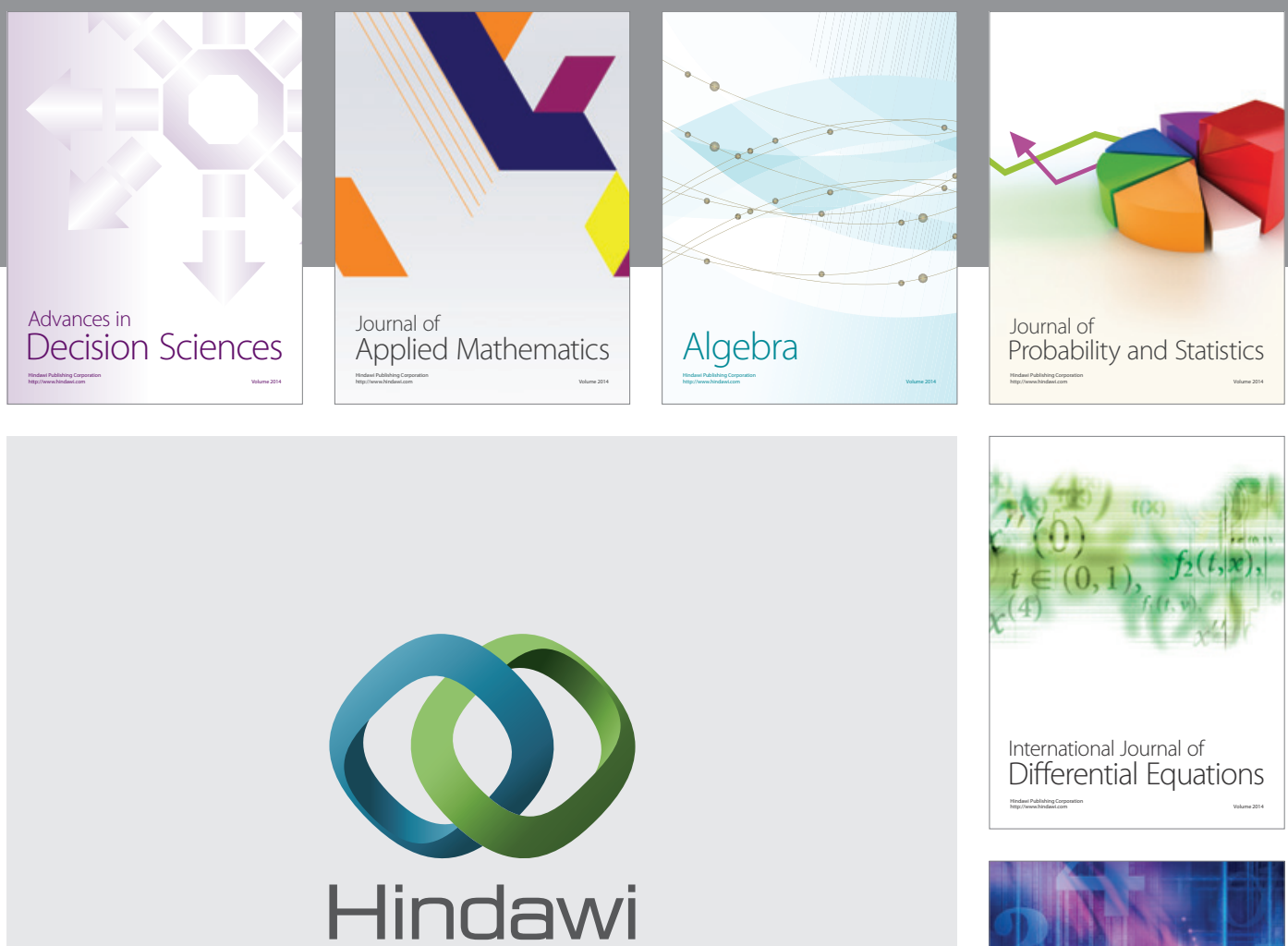

Submit your manuscripts at http://www.hindawi.com
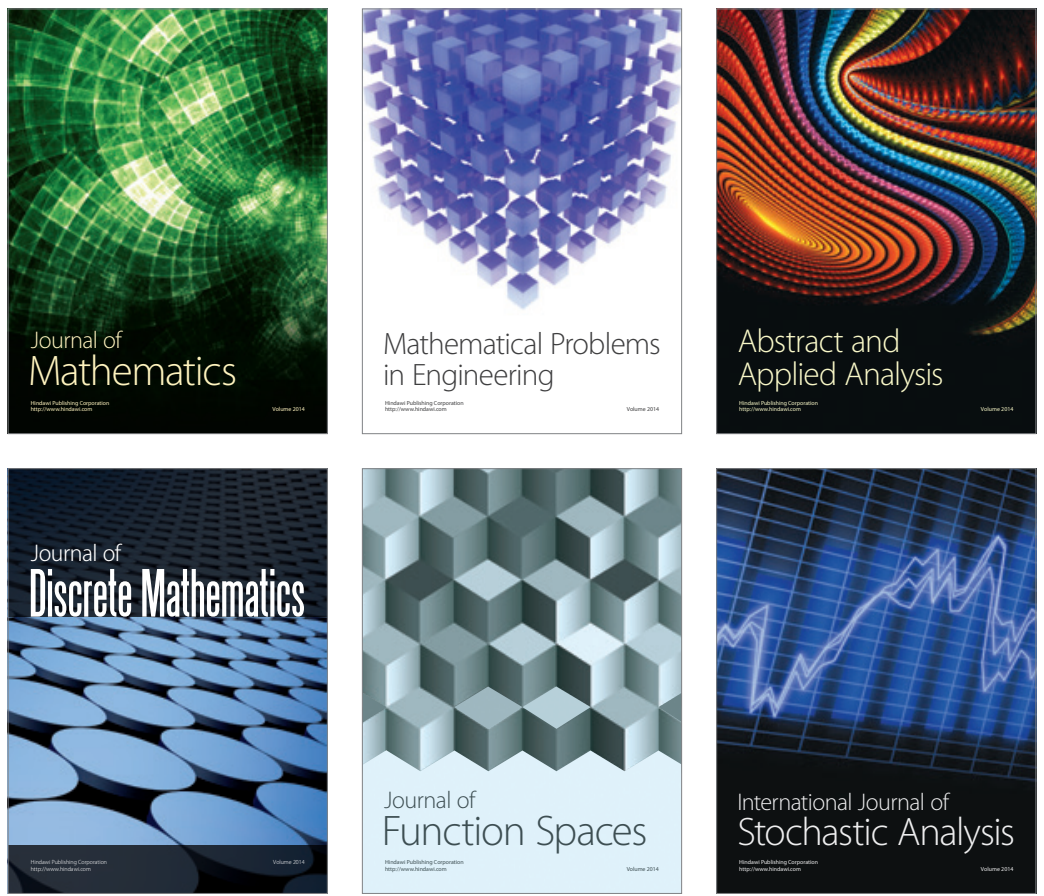

Journal of

Function Spaces

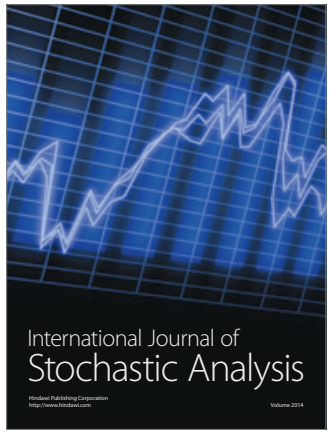

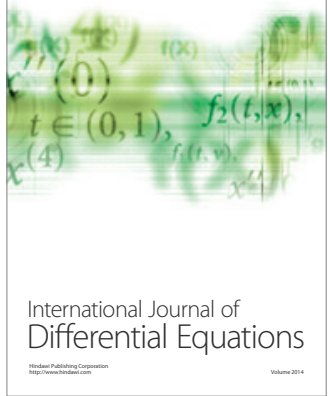
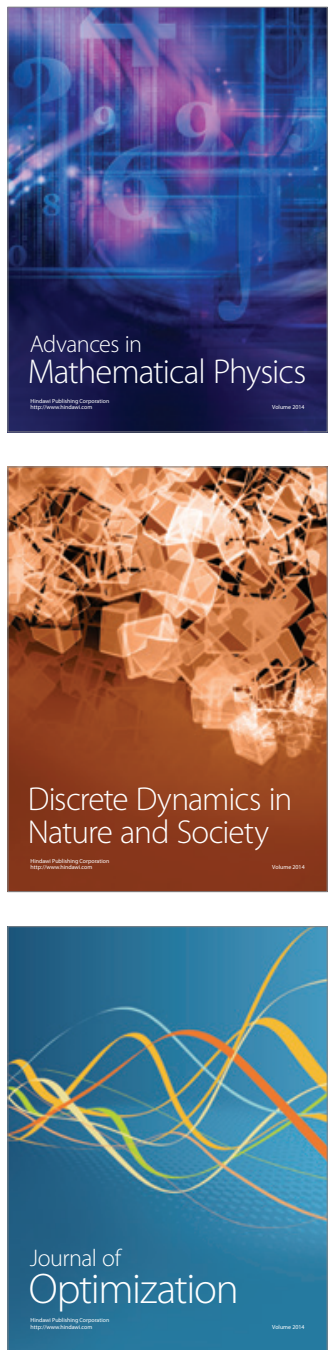